\title{
Belastende Diagnose: zwischen Erschütterung und Entlastung
}

\section{Ursula Steiner-König}

Dr. med., FMH-Delegierte in das Zentralkomitee des Tag der Kranken

\section{Ein Blick zurück}

Sind nur die betroffenen Kranken erschüttert oder geht es auch den behandelnden Ärztinnen und Ärzten (manchmal) so? - Ich erinnere mich, dass in den Siebzigerjahren der Onkologe Gerd Nagel den Basler Medizinstudenten Vorlesungen über «Unheilkunde» hielt. Damals eine Innovation, war es doch lange Zeit ungern gesehen, den Patientinnen und Patienten die Wahrheit zu sagen, ganz besonders wenn es um todbringende Diagnosen ging. Am meisten fürchtete man sich vor den Krebsdiagnosen. Die Ärzteschaft traute den gewöhnlichen Sterblichen nicht zu, mit der Konfrontation der Endlichkeit des Lebens zurechtzukommen. Heute darf die Frage gestellt werden: Waren es die Kranken oder die Ärzte, die sich damit schwertaten? Wenige Jahre später sollte die Bedrohung durch Aidserkrankungen noch wesentlich schwierigere Situationen heraufbeschwören, weil die Medizin der neu aufgetauchten Krankheit noch hilflos gegenüberstand.

\section{Die Betreuung unheilbar Kranker fordert viele im Gesundheitswesen Tätige heraus.}

Seither hat sich vieles entwickelt: Einerseits erschienen zunehmend Publikationen zum Thema der Sterbebegleitung, im Wesentlichen initiiert durch Elisabeth Kübler-Ross; On Death and Dying. Interviews mit Sterbenden (Englisch, erschienen 1969). Ein wichtiges Anliegen dieser Schweizer Pionierin der Sterbeforschung war, dass der Tod zum Leben und in die Lebensgeschichte jedes Einzelnen gehöre. In der Gesellschaft dürfen Fragen zum Sinn von Lebensverlängerung um jeden Preis nicht tabuisiert werden.

Angesichts der stetig steigenden Kosten kam dann im Gesundheitswesen die Diskussion um Rationierung und Rationalisierung aufs Tapet.

Andererseits eröffneten sich mit dem Aufkommen des Internets dem medizinischen Laien bisher ungeahnte Möglichkeiten, sich über eigene Krankheitssymptome, Diagnosen und Behandlungsmöglichkeiten zu informieren, ohne auf ein ärztliches Gegenüber angewiesen zu sein.

\section{Heutige Situation}

In Zusammenhang mit Aspekten der Qualitätsoptimierung und der Rationalisierung medizinischer Behandlungsmassnahmen verlagert sich das Schwergewicht der Betrachtung immer mehr auf Diagnose, Therapieschemata und Behandlungsprotokolle; Wirtschaftlichkeitskriterien und allfällige Limitierungen werden von Gesundheitsökonomen und Krankenversicherern ins Zentrum gerückt. Das führt dazu, dass sich der Dialog zwischen Behandelnden und Kranken immer stärker auf die Krankheiten als solche bezieht. Die Patientinnen und Patienten sehen sich dank Internet halbwegs als Fachleute für diagnostische Fragestellungen. In Sachen Therapie wären sie es gerne auch, allerdings mit grösserem Risiko der Selbstüberschätzung oder der überdimensionierten Erwartungen. Nicht von ungefähr entsteht die Tendenz, von Konsumenten und nicht mehr von Patienten zu sprechen. Zudem besteht die Gefahr, dass sich durch vermehrten Einsatz des Tiers garant der Krankenversicherer in den Behandlungsvertrag zwischen Arzt und Patient einmischt und zu seinen eigenen Gunsten zu intervenieren versucht.

Aber wo bleibt unter diesem Blickwinkel der Mensch, die einzelne Person, die an der Krankheit leidet? Die Kranken möchten in Situationen der Erschütterung doch wohl mehr als nur eine rationale Diskussion, selbst wenn ihnen das nicht immer bewusst ist. In den Spitälern ist es angesichts der Sparmassnahmen einerseits und dem Diktat der DRGs und Fallpauschalen andererseits kaum mehr möglich, sich dieser Fragestellung so zuzuwenden, dass die ArztPatienten-Beziehung adäquat zum Tragen kommt. Seitens der Behandelnden wird aufgeteilt: Beispielsweise stellt sich die Frage, ob der Kranke noch ein Akutpatient ist oder in eine Rehabilitations- oder Pflegeeinheit gehört; oder ob es einen Psychiater, Psychologen oder Sozialarbeiter braucht, um dem Mitteilungsbedürfnis des Kranken gerecht zu werden. Oder: Wer kümmert sich um die Angehörigen und ihre Anliegen? Was gehört in den Kompetenzbereich der Pflege, was in denjenigen von Physiooder Ergotherapie oder weiterer Berufsgruppen? 


\section{Ausblick}

Im Zug der zeitbedingten Veränderungen des medizinischen Krankenguts müssen etwa die Bereiche von Demenzformen, chronischen Erkrankungen oder Palliativmedizin neu gewichtet werden, da sie mengenmässig zunehmen. Die Betreuung unheilbar Kranker fordert viele im Gesundheitswesen Tätige heraus. Palliative Care und - als neues Arbeitsfeld Spiritual Care sollten oft vernachlässigte Bedürfnisse der Kranken abdecken können. Der Bedarf an entsprechenden Fachkräften nimmt zu. Man kann eine weitere Fraktionierung von Verantwortlichkeiten bedauern oder aber sie als Herausforderung annehmen. Vermutlich besteht ja ein innerer Zusammenhang zwischen der zunehmenden Technisierung einerseits und dem Bedürfnis nach einem Mehr, das mit Sinngebung zu tun hat. Wenn wir Ärztinnen und Ärzte uns weiterhin auch dem Sinngebenden verpflichtet fühlen, muss ja in der Umsetzung nicht unbedingt aufgetrennt werden, was theoretisch unterschieden wird. Das Selbstverständnis der Ärztin/ des Arztes widersteht so der Fraktionierung des eigenen Selbstbildnisses.

An der Basler Universität wird ab kommendem Frühjahr ein berufsbegleitender Master-Studiengang (Advanced Studies) «Spiritual Care» angeboten, der «befähigt zur Einbeziehung der spirituellen Dimension beim Umgang mit Krankheit und Gesundheit, Sterben und Tod» (siehe www.uniweiterbildung.ch). Im bisher Gesagten schwingt mit, dass bei «belastenden Diagnosen» eben auch eine Erschütterung aufseiten der Behandelnden zum Ausdruck kommen kann. Um den betroffenen Kranken Hilfe anbieten zu können, braucht es eine vorausgehende Selbstsorge, womit etwa das eigene Akzeptieren von Endlichkeit des Lebens sowie das Anerkennen von Medizin als Kunst und nicht als blosses Handwerk gemeint ist. Solche Haltungen befreien von übertriebenen Erwartungen sowohl bei sich selber als auch bei den uns anvertrauten Kranken.

\section{Was braucht es im Augenblick der Konfrontation des Arztes mit dem betroffenen Kranken?}

Kennen wir diesen Menschen gut genug?: In der Praxis konstelliert sich dieser Aspekt sehr unterschiedlich, je nach Alter der/des Kranken, Dauer der Behandlung und Art der bisher zur Diskussion stehenden Erkrankungen. Angesichts mannigfach begründeter zeitlicher Beschränkungen stellt sich die Frage, ob bisher Krankheiten oder ein Mensch zu behandeln waren. Ein Kollege drückte es einmal so aus: «Jener Arzt war ein ausgezeichneter Verwalter der Krankheit, aber ...» Was offenbar vermisst wurde, war das Einfühlungsvermögen und die menschliche Anteilnahme und Begleitung, als es eigentlich um eine $\mathrm{Pa}$ tientin mit einer sehr belastenden onkologischen Diagnose gegangen war.

Bin ich überhaupt in der Lage, dieser Situation ins Auge zu schauen?: Je nach eigenen Lebenserfahrungen - sei es im Familien- oder Freundeskreis oder aus der Praxistätigkeit - und Alter stehen wir Fragen nach der Endlichkeit unterschiedlich gegenüber. Vom anfänglichen Erschrecken setzt sich die Entwicklung fort hin zu zunehmender Vertrautheit mit dem Thema. Was wird beim Auffangen der Reaktionen meines Gegenübers bei mir selber ablaufen? Diese Ungewissheit stellt vielleicht die grösste Versuchung dar, das Gespräch mit der kranken Person sich nicht in allzu grosse Tiefe hinein entwickeln zu lassen. Es sei denn, die eigenen Reaktionen würden unbeachtet unterdrückt. Wie steht es punkto eigener Kompetenz?: Je nach eigener Spezialisierung ist auch dieser Aspekt gewiss unterschiedlich ausgeprägt: In der Onkologie verhält es sich anders als etwa in der Orthopädie. Letztlich jedoch ist keine Disziplin verschont. Hausärztinnen und Hausärzte dürften das bejahen. Sind wir öfters mit einer erschütternden Situation konfrontiert, wird die eigene Erschütterung geringer sein. Auch unterscheidet sich die Mitteilung, dass es sich um eine lebenslänglich begleitende chronische Erkrankung handle, von einer, bei der es um Leben oder Tod, um gewaltige Eingriffe in die bisherige Lebensweise meines Gegenübers geht. Allerdings können auch chronische Erkrankungen die Lebensweise oder Zukunftsperspektiven eines Menschen erheblich beeinflussen. Aber Hoffnung auf ein trotz allem sinnvolles Dasein kann wohl schneller zurückkehren. So kann ärztliche Kunst überleben und, zum Wohl unserer Patientinnen und Patienten, andauern.

\section{Ein Postskriptum}

Da gerade während der Niederschrift dieses Beitrags zum Tag der Kranken in unserer Ärztezeitung das Grundlagenpapier der DDQ «Shared Decision Making - Arzt und Patient entscheiden gemeinsam» [1] erschienen ist, verzichte ich auf weitere Ausführungen, um nicht «Eulen nach Athen» zu tragen. Hingegen beglückwünsche ich unsere FMH, insbesondere die Verfassenden des Artikels, zu dessen Erscheinen!

1 Gerber M, Kraft E, Bosshard C. Shared Decision Making - Arzt und Patient entscheiden gemeinsam. Schweiz Ärztezeitung. 2014;95(50):1883-9. 